\title{
Hábitos, Preferencias y Satisfacción Sexual en Estudiantes Universitarios
}

\author{
Beatriz Navarro Bravoa,b, Laura Ros Seguraa, José Miguel Latorre Postigoa, \\ José Carlos Escribano Villafruelac, Verónica López Honrubiac ${ }^{c}$, María Romero Marchante ${ }^{c}$
}

\author{
a Departamento de Psicología, \\ Facultad de Medicina de \\ Albacete (UCLM), España. \\ b Unidad de Investigación, \\ Gerencia de Atención Primaria \\ de Albacete (SESCAM), \\ España. \\ ${ }^{c}$ Alumno/a de Medicina, \\ Facultad de Medicina de \\ Albacete (UCLM), España.
}

Correspondencia: Beatriz Navarro Bravo, Facultad de Medicina, c/ Almansa, $\mathrm{n}^{\circ}$ 14, 02008 - Albacete, España. Correo electrónico: Beatriz. Navarro@uclm.es

Recibido el 31 de agosto de 2010.

Aceptado para su publicación el 24 de septiembre de 2010.

\section{RESUMEN}

Objetivo. Aproximarnos a las costumbres, preferencias y satisfacción sexual de una muestra de jóvenes universitarios.

Diseño. Estudio observacional de carácter transversal.

Emplazamiento. El estudio se llevó a cabo en el contexto universitario de Castilla-La Mancha durante el curso 2009/10.

Participantes. Participaron 199 estudiantes universitarios con edades comprendidas entre los 18-29 años.

Mediciones principales. Se recogieron datos sobre las costumbres sexuales de los participantes, sus preferencias, la satisfacción con su vida sexual y sus datos sociodemográficos.

Resultados. La edad media de inicio de las relaciones sexuales es 17,3 años. El $51,9 \%$ de los participantes tienen relaciones sexuales entre 3 y 10 veces al mes, aunque en el caso de aquellos sin pareja estable esta frecuencia es menor. Mediante la escala de satisfacción sexual ISS se ha determinado que los participantes están satisfechos con sus relaciones sexuales, y que no existen diferencias estadísticamente significativas en la satisfacción sexual entre hombres (media: 20,1 ) y mujeres (media: 16,8 ), $\mathrm{t}(121)=1,62, \mathrm{p}=0,11$. El $77,1 \%$ de la muestra dice alcanzar el orgasmo siempre o casi siempre. La postura preferida para los hombres es la relación sexual con el hombre detrás de la mujer, mientras que las mujeres prefieren estar colocadas encima del hombre. El preservativo masculino es el anticonceptivo más habitual y sólo el 1,1\% de la muestra no utiliza habitualmente métodos anticonceptivos.

Conclusiones. La edad de la primera relación sexual tiende a disminuir y las personas con pareja estable tienen una mayor frecuencia de relaciones sexuales. Tanto hombres como mujeres se muestran satisfechos con su vida sexual e informan de una elevada frecuencia de orgasmos en sus relaciones sexuales. Existe un elevado uso de anticonceptivos por parte de los jóvenes.

Palabras clave. Conducta Sexual, Primera Relación Sexual, Satisfacción Sexual, Métodos Anticonceptivos.

\section{ABSTRACT}

Habits, Preferences and Sexual Satisfaction in University Students

Objective. To investigate the sexual habits, preferences and satisfaction of a sample of young university students.

Design. Cross-sectional, observational study.

Setting. Performed in a university setting in Castilla-La Mancha during 2009/10.

Participants. One hundred and ninety-nine university students aged between 18-29 years.

Main Measurements. Data were collected on the sexual habits of the participants, their preferences, satisfaction with their sexual life and sociodemographic data.

Results. The mean age at first sexual relation was 17.34 years. Fifty-two percent of participants had sexual relations between 3 and 10 times a month, although in those without a stable partner this frequency was lower. Using the index of sexual satisfaction (ISS) it was found that participants are satisfied with their sexual relations, and that sexual satisfaction did not differ between men (Mean: 20.07) and women (Mean: 16.79), $t(121)=1.62, p=0.11$. Seventy-seven percent of the sample reported achieving orgasm always or almost always. The preferred position for males was sexual intercourse from behind the female, whereas women tended to prefer being on top of the man. The condom was found to be the most common form of contraception and only $1.1 \%$ of the sample reported not usually using contraceptive methods.

Conclusions. Age of first sexual relation appears to be decreasing and those people with a stable relationship have a higher frequency of sexual relations. Both men and women appear to be satisfied with their sexual lives and report a high frequency of orgasm. It was found that contraceptive use is high among young people in the study population.

Key words. Sexual Behaviour, First Sexual Intercourse, Sexual Satisfaction, Contraceptive Methods. 


\section{INTRODUCCIÓN}

Según el informe Durex 07/08 sobre bienestar sexual, el sexo es considerado como una actividad agradable, divertida y sumamente importante por 3 de cada 5 personas ${ }^{1}$, por tanto no debemos subestimar el peso que éste tiene en nuestra sociedad.

La Encuesta de Salud y Hábitos Sexuales de 2003, realizada en población española entre 18 y 49 años, revela que la edad media de la primera relación sexual es de 18,6 años, (18,1 hombres y $19,1$ en mujeres $)^{2}$, siendo esta edad superior a la de la mayoría de países europeos. En un estudio realizado en los Estados Unidos se determinó que la edad media de inicio en las relaciones sexuales entre los estudiantes de instituto estuvo entre los 16,4 y los 16,5 años $^{3}$, mientras que en otra investigación posterior aparece una edad media de inicio de 14,9 años en una muestra de chicas de entre 14 y 21 años $^{4}$. En el caso de España, en el grupo con edades comprendidas entre 18 y 29 años, la edad de inicio de relaciones sexuales es de 17,8 años, (17,5 en varones y 18,2 en mujeres), declarando haber tenido su primera relación antes de los 16 años el $11,4 \%$ de las mujeres y el $18,4 \%$ de los hombres $^{2}$, y siendo este porcentaje más reducido para los que cuentan con estudios universitarios.

Respecto al número de parejas sexuales a lo largo de la vida en personas de 18-29 años, el 12,3\% dice no haber tenido ninguna, el $31,6 \%$ ha tenido una, el $15,8 \%$ ha tenido dos, el $19,2 \%$ ha tenido tres o cuatro parejas, el $11,3 \%$ entre cinco y nueve y el $9,8 \%$ diez o más parejas sexuales. La mayoría de este grupo ha tenido una única pareja sexual en los últimos 12 meses $(59,4 \%)$, siendo la media de parejas sexuales en este mismo periodo de 2,8 en los hombres y de 1,4 en las mujeres. En el caso de los que tienen estudios universitarios, el $19,4 \%$ de los hombres ha tenido relaciones sexuales con una sola persona en su vida, sucediendo esto mismo en el $49,0 \%$ de las mujeres. En Castilla-La Mancha más del $30 \%$ de los hombres y más del $65 \%$ de las mujeres declaran haber tenido una única pareja sexual durante toda su vida ${ }^{2}$. En muestra no española se ha visto que las jóvenes de entre 14 y 21 años refieren haber tenido una única pareja en el $21 \%$ de los casos, 2 parejas en el $19 \%$ y 3 o más parejas en el $60 \% 4$.

En lo relativo a la frecuencia de relaciones sexuales, en España existe un promedio anual de 118 (superior a la media global que es de 103), lo que equivaldría a 9,8 veces al mes. El $66 \%$ de los es- pañoles siempre o casi siempre alcanza el orgasmo, frente a la media mundial del $48 \%$. En España los hombres consiguen el orgasmo en el $81 \%$ de las ocasiones y las mujeres en el $52 \%{ }^{1}$.

Respecto al uso de anticonceptivos, en una encuesta realizada a estudiantes de medicina se observó que son el preservativo y los anticonceptivos orales los métodos más utilizados ${ }^{5}$. El uso del preservativo está bastante extendido en España entre las personas de entre 18 y 29 años. El 79,6\% lo usaron en su primera relación, porcentaje muy superior al grupo de 40 o más años, que dijeron haberlo utilizado el $31,5 \%$. El $63,0 \%$ de los hombres y el $61,9 \%$ de las mujeres entre 18 y 29 años han utilizado sistemáticamente preservativo con las parejas ocasionales de los últimos 12 meses. Respecto a quién se encarga del preservativo en la primera relación con nuevas parejas, son los hombres los que suelen tomar la iniciativa, en casi 3 de cada 4 ocasiones el preservativo lo llevaba el hombre ${ }^{2}$. A pesar de que el uso del preservativo es bastante común entre los jóvenes, existen diferentes circunstancias que pueden hacer que éste se reduzca, pues se ha visto que existe una correlación significativa entre el consumo de alcohol y el comportamiento sexual de riesgo de no usar preservativo ${ }^{6}$ y que las personas que consumen alcohol en exceso presentan un mayor riesgo de incurrir en conductas sexuales que pueden llevar a infecciones por $\mathrm{VIH}^{7}$.

La práctica sexual preferida por los hombres es el sexo vaginal con la mujer encima, seguido por la postura con entrada posterior. En cambio, las mujeres prefieren el cunnilingus y, en segundo lugar, la mujer encima. La práctica menos elegida por ambos es el sexo anal ${ }^{8}$. En el estudio de Auslander et al. se ha determinado que el $54 \%$ de las chicas entre 14 y 21 años ha tenido sexo vaginal y oral, mientras que el $13 \%$ ha practicado sexo vaginal, oral y anal ${ }^{4}$.

Sobre otras costumbres sexuales, un $3,9 \%$ de los hombres y un $2,7 \%$ de las mujeres manifiestan haber tenido relaciones homosexuales en algún momento de su vida (el $1,1 \%$ de los hombres y el $0,2 \%$ de las mujeres ha tenido únicamente relaciones homosexuales), siendo el $96,7 \%$ de los encuestados los que han tenido relaciones sexuales exclusivamente con personas del sexo opuesto. El 2,8\% de los hombres ha tenido relaciones con ambos sexos, ocurriendo esto mismo en el $2,5 \%$ de las mujeres ${ }^{2}$.

Por último, respecto a los niveles de satisfacción sexual, en nuestro país cerca de la mitad de la 
población se declara satisfecha con su vida sexual ( $44 \%$ de los hombres y $54 \%$ de las mujeres). Hasta los 25 años los hombres presentan mayor grado de satisfacción, momento a partir del cual son superados por las mujeres, aunque en ambos va disminuyendo con la edad ${ }^{1}$. En el estudio de Santos-Iglesias, por otro lado, no se encontraron diferencias significativas entre hombres y mujeres en los niveles de satisfacción sexual evaluados mediante el Índice de Satisfacción Sexual (ISS) ${ }^{9}$.

El objetivo principal de este estudio es aproximarnos a las costumbres y preferencias sexuales de una muestra de jóvenes universitarios, así como evaluar el nivel de satisfacción de éstos con su vida sexual.

\section{MATERIAL Y MÉTODO}

Se trata de un estudio observacional de carácter transversal en estudiantes universitarios de CastillaLa Mancha, realizado durante el curso 2009/10. Se recogieron datos sobre la experiencia sexual de los participantes: si habían tenido relaciones sexuales, la edad de la primera relación y el número de parejas sexuales a lo largo de la vida y en el último año; sobre sus hábitos: frecuencia de relaciones sexuales, quién toma la iniciativa, relaciones sexuales después de haber consumido alcohol, uso de anticonceptivos, uso de material pornográfico y objetos de sex-shop, y relaciones sexuales con personas del propio sexo; sobre sus preferencias sexuales y sobre su nivel de satisfacción sexual: frecuencia de orgasmo, valoración de diferentes prácticas sexuales, satisfacción con determinados aspectos de la vida sexual y satisfacción sexual evaluada mediante la escala Índice de Satisfacción Sexual (ISS) ${ }^{10}$. Así mismo, se les encuestó sobre sus características sociodemográficas.

Se propuso la participación a 232 personas, de las que finalmente respondieron a la encuesta 199 (tasa de respuesta del $85,78 \%$ ). Los criterios de inclusión fueron tener una edad entre 18 y 29 años, ser estudiante universitario y aceptar la participación en el estudio. La muestra estuvo compuesta por 74 hombres $(37,2 \%)$ y 125 mujeres $(62,8 \%)$ con edades comprendidas entre 18 y 29 años, y con una edad media de 21,6 años (DE = 1,8). Todos ellos eran estudiantes universitarios en el momento de participar en el estudio, la mayoría de ellos de las titulaciones de Medicina (39,2\%), Magisterio (15,6\%) y Administración y Dirección de Empresas (10,6\%).

Se elaboró un cuaderno de recogida de datos específicamente diseñado para el estudio. Para la elaboración del cuestionario y la obtención de datos se contó con la colaboración de los alumnos de la asignatura de Sexología Médica de la Facultad de Medicina de Albacete durante el curso 2009/10. Se solicitó su colaboración voluntaria en el estudio, pidiendo a cada uno de ellos que intentase conseguir al menos 4 encuestas contestadas seleccionando a los participantes entre las personas de su entorno.

\begin{tabular}{l|ccc}
\hline & $\begin{array}{c}\text { Muestra total } \\
(\mathbf{n = 1 8 4})\end{array}$ & $\begin{array}{c}\text { Hombre } \\
\mathbf{( n = 6 9 )}\end{array}$ & $\begin{array}{c}\text { Mujer } \\
(\mathbf{n}=\mathbf{1 1 5})\end{array}$ \\
\hline Frecuencia de las relaciones sexuales & & & \\
$\quad$ Ninguna vez al mes & $7,1 \%$ & $11,8 \%$ & $4,3 \%$ \\
$\quad$ Entre 1 y 2 veces al mes & $16,9 \%$ & $13,2 \%$ & $19,1 \%$ \\
$\quad$ Entre 3 y 5 veces al mes & $23,5 \%$ & $23,5 \%$ & $23,5 \%$ \\
Entre 6 y 10 veces al mes & $28,4 \%$ & $30,9 \%$ & $27,0 \%$ \\
Entre 10 y 15 veces al mes & $12,6 \%$ & $10,3 \%$ & $13,9 \%$ \\
Más de 15 veces al mes & $11,5 \%$ & $10,3 \%$ & $12,2 \%$ \\
& & & \\
Toma de iniciativa en las relaciones sexuales & & & \\
$\quad$ Nunca & $0 \%$ & $0 \%$ & $11,5 \%$ \\
Casi nunca & $9,8 \%$ & $7,1 \%$ & $67,3 \%$ \\
De vez en cuando & $53,0 \%$ & $30,0 \%$ & $19,4 \%$ \\
Casi siempre & $30,1 \%$ & $47,2 \%$ & $1,8 \%$ \\
Siempre & $7,1 \%$ & $15,7 \%$ & \\
\hline
\end{tabular}

Tabla 1. Frecuencia de hábitos sexuales. 


\begin{tabular}{|c|c|c|c|}
\hline & \multirow{2}{*}{$\begin{array}{l}\text { Muestra total } \\
\qquad(n=184)\end{array}$} & \multicolumn{2}{|c|}{ Género } \\
\hline & & $\begin{array}{l}\text { Hombre } \\
(n=69)\end{array}$ & $\begin{array}{c}\text { Mujer } \\
(n=115)\end{array}$ \\
\hline Normalmente no utilizo ningún método anticonceptivo & $1,1 \%$ & $1,4 \%$ & $0,9 \%$ \\
\hline Preservativo masculino & $67,8 \%$ & $72,9 \%$ & $64,6 \%$ \\
\hline Preservativo masculino y píldora anticonceptiva & $10,9 \%$ & $10,0 \%$ & $11,5 \%$ \\
\hline Píldora anticonceptiva & $4,9 \%$ & $2,9 \%$ & $6,2 \%$ \\
\hline Preservativo masculino y femenino & $1,6 \%$ & $0 \%$ & $2,7 \%$ \\
\hline Preservativo masculino y marcha atrás & $3,3 \%$ & $4,3 \%$ & $2,7 \%$ \\
\hline Preservativo masculino y anillo vaginal & $1,6 \%$ & $1,4 \%$ & $1,8 \%$ \\
\hline Preservativo masculino, píldora anticonceptiva y marcha atrás & $1,6 \%$ & $1,4 \%$ & $1,8 \%$ \\
\hline Otros & $7,2 \%$ & $5,7 \%$ & $7,8 \%$ \\
\hline
\end{tabular}

Tabla 2. Frecuencia de uso de los diferentes métodos anticonceptivos.

\begin{tabular}{l|ccc}
\hline & $\begin{array}{c}\text { Muestra total } \\
(\mathbf{n = 1 9 9 )}\end{array}$ & $\begin{array}{c}\text { Hombre } \\
(\mathbf{n = 7 4 )}\end{array}$ & $\begin{array}{c}\text { Mujer } \\
(\mathbf{n = 1 2 5})\end{array}$ \\
\hline Nunca ha consumido & $45,9 \%$ & $9,5 \%$ & $67,8 \%$ \\
Películas alquiladas y/o compradas & $6,7 \%$ & $11,0 \%$ & $4,1 \%$ \\
Películas obtenidas en internet & $39,7 \%$ & $72,6 \%$ & $19,8 \%$ \\
Imágenes en revistas & $2,1 \%$ & $1,4 \%$ & $2,5 \%$ \\
Imágenes obtenidas en internet & $4,1 \%$ & $5,5 \%$ & $3,3 \%$ \\
Otros & $1,5 \%$ & $0 \%$ & $2,5 \%$ \\
\hline
\end{tabular}

Tabla 3. Consumo de material pornográfico.

\begin{tabular}{|c|c|c|}
\hline & $\begin{array}{l}\text { Hombre } \\
(\mathrm{n}=74)\end{array}$ & $\begin{array}{c}\text { Mujer } \\
(n=125)\end{array}$ \\
\hline Sólo con mujeres & $89,1 \%$ & $0 \%$ \\
\hline Más a menudo con mujeres pero al menos en una ocasión con un hombre & $1,4 \%$ & $0 \%$ \\
\hline Igual con hombres que con mujeres & $0 \%$ & $0,8 \%$ \\
\hline Más a menudo con hombres pero al menos en una ocasión con una mujer & $1,4 \%$ & $4,0 \%$ \\
\hline Sólo con hombres & $6,7 \%$ & $92,0 \%$ \\
\hline Nunca he tenido contacto sexual de ningún tipo. & $1,4 \%$ & $3,2 \%$ \\
\hline
\end{tabular}

Tabla 4. Género del compañero en los contactos sexuales.

\begin{tabular}{l|ccc}
\hline & Muestra total & \multicolumn{2}{c}{ Género } \\
\cline { 3 - 4 } & $(\mathbf{n = 1 8 4 )}$ & $\begin{array}{c}\text { Hombre } \\
(\mathbf{n = 6 9 )}\end{array}$ & $\begin{array}{c}\text { Mujer } \\
(\mathbf{n}=\mathbf{1 1 5})\end{array}$ \\
\hline Felación & $6,31(3,25)$ & $8,30(2,07)$ & $5,09(3,25)$ \\
Cunnilingus & $6,97(3,00)$ & $6,63(2,68)$ & $7,17(3,17)$ \\
Relación sexual con la mujer encima & $8,05(1,92)$ & $8,08(1,58)$ & $8,03(2,12)$ \\
Relación sexual con el hombre colocado detrás de la mujer & $7,45(2,39)$ & $8,40(1,68)$ & $6,87(2,58)$ \\
Postura del misionero (hombre encima) & $7,75(1,76)$ & $7,51(1,75)$ & $7,91(1,76)$ \\
Sexo anal & $1,58(2,87)$ & $2,89(3,61)$ & $0,90(2,12)$ \\
\hline
\end{tabular}

Tabla 5. Media y (desviaciones típicas) de preferencias sobre diferentes prácticas sexuales. 
Estos alumnos recibieron instrucciones en varias sesiones y también material escrito sobre cómo realizar las encuestas, con el fin de que la recogida de datos se realizase de una forma homogénea. Después de esto se les facilitaron varias copias de la encuesta en papel junto con las hojas de información al participante, dándoles un plazo de 2-3 semanas para devolver las encuestas cumplimentadas. Las encuestas se realizaron de forma autoadministrada.

Después de la recogida de datos se creó una base de datos con el programa SPSS v.15.0. La información fue revisada para detectar posibles errores y depurar la base antes de realizar el análisis estadístico. En primer lugar se realizó un análisis descriptivo de los datos, para posteriormente realizar diversas comparaciones de medias y proporciones en función del tipo de variable, comparando los resultados obtenidos por los participantes en función del sexo y de si tenían o no pareja estable en el momento de realización del estudio.

\section{RESULTADOS}

En total se obtuvieron las encuestas de 199 participantes (74 hombres y 125 mujeres) con una edad media de 21,6 años (DE: 1,8), de los cuales el $65,3 \%$ tenía pareja estable cuando se procedió a la encuesta.

Para el análisis de los hábitos, las preferencias y la satisfacción sexual de los participantes, se tomaron en consideración únicamente las encuestas de aquellas personas que habían mantenido en alguna ocasión relaciones sexuales coitales (184 personas). En relación con la primera relación sexual, encontramos que la edad media de inicio es 17,3 años ( $D E=1,7)$ y que esta edad no varía en función del sexo: 17,3 años (DE: 1,8) en los hombres y 17,4 años $(\mathrm{DE}: 1,65)$ en las mujeres. Además, el $39,8 \%$ de los participantes $(39,4 \%$ de los hombres y $40,0 \%$ de las mujeres) se muestra bastante o totalmente satisfecho con la edad de su primera relación sexual.

Respecto al número total de parejas que han tenido los participantes en el estudio, encontramos que el $69,7 \%(67,1 \%$ de los hombres y $71,0 \%$ de las mujeres) han tenido entre 1 y 3 parejas sexuales a lo largo de su vida y que en el último año lo más habitual ha sido tener una única pareja sexual $(70,7 \%$ del total de la muestra, $69,6 \%$ de los hombres y $71,3 \%$ de las mujeres).
La tabla 1 muestra los resultados sobre la frecuencia de realización de conductas sexuales. En ella observamos que el $51,9 \%$ de los participantes tiene relaciones sexuales entre 3 y 10 veces al mes, excepto aquellos que no tienen pareja estable, donde el $50,9 \%$ de las personas mantienen una frecuencia de relaciones sexuales de 1 a 5 veces al mes. Además, son los hombres los que siempre o casi siempre toman la iniciativa sexual $(62,9 \%)$, mientras que las mujeres lo suelen hacer casi nunca $o$ sólo de vez en cuando $(78,8 \%)$.

Respecto a los índices de satisfacción con la frecuencia de las relaciones sexuales, encontramos que el $53,8 \%$ de la muestra $(49,3 \%$ de los hombres y $56,5 \%$ de las mujeres) se declara bastante o totalmente satisfecho. La satisfacción en relación con la calidad de las relaciones sexuales también es elevada en el $84,4 \%$ de los participantes $(81,7 \%$ de los hombres y $85,9 \%$ de las mujeres). Del mismo modo, el $69,9 \%$ de los participantes $(67,6 \%$ de los hombres y $71,3 \%$ de las mujeres) se considera bastante o totalmente satisfecho con la variedad de sus relaciones sexuales. Por otro lado, los resultados obtenidos con la escala de satisfacción sexual ISS señalan que los participantes en general están satisfechos con sus relaciones sexuales (media: 18,0; DE: 10,9) y que no existen diferencias estadísticamente significativas en la satisfacción sexual entre hombres (media: 20,1; DE: 11,7) y mujeres (media: 16,8; DE: 10,3), $\mathrm{t}(121)=1,62, \mathrm{p}=0,11$.

Centrándonos en los aspectos relacionados con la consecución de orgasmos, encontramos que, en general, el $7,1 \%$ de los participantes tiene dificultades para alcanzar el orgasmo, mientras que el $15,8 \%$ lo alcanza de vez en cuando y el $77,1 \%$ lo consigue siempre o casi siempre. Si estudiamos la frecuencia de orgasmos en función del sexo, encontramos que las hombres consiguen llegar al orgasmo más frecuentemente: el $87,1 \%$ de los hombres consigue alcanzar el orgasmo siempre o casi siempre frente a un $71 \%$ de las mujeres $\left(\chi^{2}=14,8\right.$, $p=0,00)$. Si analizamos el índice de satisfacción con la consecución de orgasmos, encontramos que el $76,7 \%$ (el $87,1 \%$ de hombres y el $70 \%$ de las mujeres) se encuentra bastante o totalmente satisfecho con la frecuencia de orgasmos en sus relaciones sexuales. En este sentido hay que señalar que son los hombres los que mayores índices de satisfacción alcanzan en comparación con las mujeres $\left(\chi^{2}=12,81, p=0,01\right)$.

Respecto al uso de anticonceptivos, encontramos que, de forma generalizada, ambos miembros de 
la pareja se encargan por igual de la consecución de los éstos $(43,2 \%$ en la muestra total, $39,1 \%$ en el grupo de hombres y el $45,6 \%$ en el grupo de mujeres). Por otro lado, en relación con la frecuencia de uso de los anticonceptivos, hemos analizado por separado su uso dentro de una relación estable o dentro de relaciones esporádicas. Así, cuando hablamos de una relación de pareja estable, encontramos que el $93,4 \%$ de la muestra total utiliza siempre o casi siempre métodos anticonceptivos y que estos porcentajes se mantienen independientemente del género: $91,3 \%$ de los hombres y $94,7 \%$ de las mujeres utilizan los anticonceptivos de forma habitual. Si nos centramos en los casos de relaciones esporádicas, los porcentajes son también muy similares: el $92,9 \%$ de todos los participantes (92,0\% de los hombres y $93,5 \%$ de las mujeres) utilizan anticonceptivos siempre o casi siempre. La tabla 2 muestra cuáles son los métodos anticonceptivos más utilizados y los resultados señalan que el preservativo masculino es el método más habitual tanto en hombres como en mujeres y que sólo el $1,1 \%$ de la muestra no utiliza habitualmente métodos anticonceptivos.

En relación con la frecuencia de relaciones sexuales tras la ingesta de alcohol, encontramos que sólo el $11 \%$ de la muestra suele consumir alcohol de forma habitual antes de mantener relaciones sexuales. Este porcentaje aumenta ligeramente en el caso de los hombres $(15,7 \%)$ o de no tener pareja estable $(16,3 \%)$, mientras que disminuye en las mujeres $(9,7 \%)$ y en las parejas estables $(10,3 \%)$, aunque estas diferencias no llegan a alcanzar significación estadística $\left(\chi^{2}=1,22, p=0,27\right.$ en la variable género y $\chi^{2}=0,17, p=0,68$ en la variable tener pareja estable).

Con esta muestra se ha analizado la frecuencia de uso de materiales pornográficos o procedentes de tiendas sex-shop. Así, encontramos que el 60,3\% de los participantes en el estudio $(63,5 \%$ de los hombres y $58,9 \%$ de las mujeres) no han utilizado nunca objetos de tiendas sex-shop. Sin embargo, en los casos en los que el participante tiene pareja estable el consumo de este tipo de objetos es mayor: el $44,5 \%$ de personas con pareja estable utilizan este tipo de material frente a sólo un $28,4 \%$ de personas sin pareja que lo utilizan $\left(\chi^{2}=4,84\right.$, $p$ $=0,03)$. La tabla 3 muestra los datos relacionados con el tipo de material erótico que utilizan los participantes del estudio. Los resultados señalan que el material más habitual son las películas obtenidas en Internet y que las mujeres son las que menos utilizan el material pornográfico, ya que el $67,8 \%$ afirma no haberlo consumido nunca frente a un $9,5 \%$ de los hombres $\left(\chi^{2}=7,13, p=0,00\right)$.

Respecto a la frecuencia de género del compañero sexual, encontramos que el contacto sexual más habitual es el de tipo heterosexual, ya que sólo el $9,5 \%$ de los hombres y el $4,8 \%$ de las mujeres han mantenido en alguna ocasión contacto sexual con alguien del mismo sexo (tabla 4).

Por último, en la tabla 5 se puede observar cuáles son las preferencias en cuanto a prácticas sexuales de los participantes del estudio. Así, en una escala de 1 a 10, observamos que los hombres y las mujeres no coinciden en la práctica preferida: a los hombres les gusta más estar colocados detrás de la mujer, mientras que las mujeres prefieren estar encima.

\section{DISCUSIÓN}

En este estudio se ha comprobado que la edad de la primera relación sexual entre los jóvenes de entre 18 y 29 años es de 17,3 años (17,3 en hombres y 17,4 en mujeres). Comparando estos resultados con otros más antiguos, se puede observar que la edad de las primeras relaciones sexuales tiende a disminuir con el paso del tiempo, como ya se ha resaltado en otros estudios ${ }^{11}$. En la Encuesta de Salud y Hábitos Sexuales de $2003^{2}$ la edad media de inicio de las relaciones sexuales fue de 17,8 años ( 17,5 en hombres y 18,2 en mujeres) para las personas con edades entre 18 y 29 años, y más elevada en el caso de las personas entre 30-39 años (18,8 años) y entre 40-49 años $(19,3)$. Respecto al número de parejas sexuales a lo largo de la vida y en el último año, los resultados también son similares a la encuesta de Salud y Hábitos Sexuales de 2003, siendo lo más habitual haber tenido una única pareja sexual en el último año.

En relación a la frecuencia de relaciones sexuales se ha visto que éstas son más habituales en personas con pareja estable. El 51,9\% de este grupo afirma tener relaciones sexuales entre 3 y 10 veces al mes. Con la cautela necesaria, teniendo en cuenta el diferente método de evaluación de este dato, los resultados se asemejan a los ofrecidos en el informe Durex, en los que se habla de una frecuencia media de relaciones sexuales de 9,8 veces al mes en población española ${ }^{1}$.

Tanto hombres como mujeres se muestran satisfechos con su vida sexual y los diferentes aspectos 
de ésta que han sido evaluados en este estudio, en consonancia con estudios anteriores que hablan de que aproximadamente el $80 \%$ de las personas se encuentran relativa o completamente satisfechas con su vida sexual ${ }^{12}$. Los resultados obtenidos con la escala de satisfacción sexual ISS se encuentran en la misma línea que los obtenidos en estudios anteriores $^{9}$, en los que tampoco aparecieron diferencias estadísticamente significativas entre los niveles de satisfacción de hombres y mujeres.

En relación a la frecuencia de orgasmos encontrada en este estudio (el $87,1 \%$ de los hombres y el $71,0 \%$ de las mujeres dicen alcanzarlo siempre o casi siempre), ésta es muy similar a la encontrada en estudios anteriores, en los que se habla de más de un $90 \%$ de hombres y cerca de un $70 \%$ de mujeres que dicen haber alcanzado el orgasmo siempre o normalmente en los últimos 12 meses $^{13-14}$.

Respecto al uso de anticonceptivos, igual que indica la bibliografía disponible, son el preservativo y la píldora anticonceptiva los métodos más comúnmente utilizados por los jóvenes ${ }^{5}$, aunque nuestros resultados difieren de los encontrados en la Encuesta Nacional de Salud y Hábitos Sexuales en relación a quién suele encargarse de éstos ${ }^{2}$. Según esta encuesta es el hombre el que se ocupa de proporcionar los anticonceptivos en la mayoría de las ocasiones cuando hablamos de la primera relación con una nueva pareja, mientras que en nuestros resultados los jóvenes han informado de que ambos sexos se ocupan por igual de esto (sin tener en cuenta si la pareja es nueva o no). También en la frecuencia de uso de preservativo en las relaciones esporádicas nuestros resultados difieren de los de la mencionada encuesta, indicando los participantes en nuestro estudio una frecuencia mucho más elevada de uso de anticonceptivos en estas ocasiones (92,9\% dijeron usar anticonceptivos siempre o casi siempre, frente al $61,9 \%$ de participantes en la encuesta del INE que dijeron haber usado sistemáticamente anticonceptivos con parejas ocasionales en los 12 meses anteriores).

La mayoría de los participantes han tenido únicamente contacto heterosexual a lo largo de sus vidas $(89,1 \%$ de los hombres y $92,0 \%$ de las mujeres), igual que sucede en la encuesta del $\mathrm{INE}^{2}$. A pesar de esto, en nuestro estudio se ha obtenido una prevalencia más elevada de personas que han mantenido en alguna ocasión contacto homosexual y de hombres que han mantenido exclusivamente contacto homosexual, llamando la atención la diferencia encontrada entre la prevalencia de contacto exclusivamente homosexual en hombres en nuestro estudio $(6,7 \%)$ frente a la de la encuesta del INE $(1,1 \%)$.

En nuestro estudio hemos encontrado que en cuanto a posturas sexuales, las mejor valoradas por los hombres fueron la del hombre colocado detrás de la mujer, la felación y la mujer colocada encima, mientras que las mejor valoradas por las mujeres fueron la de la mujer colocada encima, la del misionero y el cunnilingus. Estos resultados, aunque no son exactamente iguales, van en la misma línea que otros más antiguos en los que se habla de que las posturas preferidas por el hombre son, por este orden, la de la mujer encima, la del hombre colocado detrás de la mujer y la felación, y las preferidas de las mujeres el cunnilingus, la mujer colocada encima y el hombre colocado detrás de la mujer ${ }^{8}$. De la misma manera que en la encuesta citada anteriormente, en nuestro estudio la práctica menos puntuada por hombres y mujeres ha sido el sexo anal.

La principal limitación de este estudio ha sido la posible heterogeneidad en la recogida de datos. Si bien se entrenó a los alumnos en el manejo del cuestionario y se les dio instrucciones precisas para homogeneizar el proceso, el hecho de que cada uno de ellos realizase la recogida de datos en su propio entorno ha podido introducir algún sesgo en esta fase del estudio. Por otro lado, las particularidades de los estudios sobre temas de sexualidad siempre plantean la posibilidad de sesgos de deseabilidad social en los participantes a la hora de ofrecer las respuestas. Como investigación futura se propone estudiar en cursos posteriores los hábitos, preferencias y satisfacción sexuales de estudiantes universitarios, utilizando una muestra más amplia y recogiendo los datos de forma grupal en las aulas universitarias.

Como conclusión, la edad de la primera relación sexual tiende a disminuir en los jóvenes de 18 a 29 años. Lo más habitual en ellos es haber tenido una única pareja sexual en el último año y las personas con pareja estable tienen una mayor frecuencia de relaciones sexuales. Tanto hombres como mujeres se muestran satisfechos con su vida sexual e informan de una elevada frecuencia de orgasmos en sus relaciones sexuales. Existe un elevado uso de anticonceptivos por parte de los jóvenes y entre éstos el más habitual es el preservativo. Sólo un $1,1 \%$ de los jóvenes dijeron no usar anticonceptivos habitualmente. Los que han tenido contacto homosexual al menos en alguna ocasión son minoría y las posturas preferidas por hombres y mujeres son 
las del sexo vaginal con el hombre colocado detrás y con la mujer colocada encima respectivamente.

\section{AGRADECIMIENTOS}

A los alumnos de la asignatura de Sexología Médica del curso 2009/10 de la Facultad de Medicina de Albacete que colaboraron en la recogida de datos y al Doctor Simon Kyle por dedicar parte de su tiempo a colaborar con este artículo.

\section{BIBLIOGRAFÍA}

1. Informe Durex sobre Bienestar Sexual-GSWS 2007/08, 2007. Disponible en: http://www.durex.com/es-es/flashrepository/documents/informe $\% 20$ durex $\% 20$ de $\% 20$ bienestar\%20sexual\%20\%20-\%20satisfacci\%C3\%B3n.pdf

2. INE Encuesta de Salud y Hábitos Sexuales 2003, 2006. Disponible en: http://www.ine.es/prodyser/pubweb/saludyhs03/saludyhs03_resu.pdf

3. Warren CW, Santelli JS, Everett SA, Kann L, Collins JL, Cassel C, et al. Sexual behavior among U.S. high school students, 1990-1995. Fam Plann Perspect. 1998; 30(4):1702.

4. Auslander BA, Biro FM, Succop PA, Short MB, Rosenthal $\mathrm{SL}$. Racial/ethnic differences in patterns of sexual behaviour and STI risk among sexually experienced adolescent girls. J Pediatr Adolesc Gynecol. 2009; 22(1):33-9.
5. Shindel AW, Ferguson GG, Nelson CJ, Brandes SB. The sexual lives of medical students: a single institution survey. J Sex Med. 2008; 5(4):796-803.

6. Richter DL, Valois RF, McKeown RE, Vincent ML. Correlates of condom use and number of sexual partners among high school adolescents. J Sch Health. 1993; 63(2):91-6.

7. Castilla J, Barrio G, Belza MJ, de la Fuente L. Drug and alcohol consumption and sexual risk behaviour among young adults: results from a nacional survey. Drug Alcohol Depend. 1999; 56:47-53.

8. Bishop C, Osthelder X. Sexualia. From Prehistory to Ciberspace. Cologne, Germany: Könemann Verlagsgesellschaft; 2001.

9. Santos Iglesias P, Sierra JC, García M, Martínez A, Sánchez A, Tapia MI. Índice de Satisfacción Sexual (ISS): un estudio sobre su fiabilidad y validez. Intern Jour Psych Psychol Ther. 2009; 9(2):259-73.

10. Crooks R, Baur K. Nuestra sexualidad. México, DF: International Thompson; 2000.

11. Hawes ZC, Wellings K, Stephenson J. First heterosexual intercourse in the United Kingdom: a review of the literature. J Sex Res. 2010; 47(2):137-52

12. Colson MH, Lemaire A, Pinton P, Hamidi K, Klein P. Sexual behaviours and mental perception, satisfaction and expectations of sex life in men and women in France. J Sex Med. 2006; 3(1):121-31.

13. Laumann EO, Gagnon JH, Michael RT, Michaels S. The social organization of sexuality: sexual practices in the United States. Chicago: University of Chicago Press; 1994.

14. Michael RT, Gagnon JH, Laumann EO, Kolata G. Sex in America: A definitive survey. Boston: Little Brown; 1994. 